\title{
A REMARK ON SCHATTEN CLASS TOEPLITZ OPERATORS ON BERGMAN SPACES
}

\author{
JORDI PAU \\ (Communicated by Richard Rochberg)
}

\begin{abstract}
We provide a new characterization (valid for all $0<p<\infty$ ) of Schatten class membership of Toeplitz operators induced by positive measures acting on weighted Bergman spaces on the unit ball. The result is new even for the case of the unit disk.
\end{abstract}

Let $\mathbb{C}^{n}$ denote the Euclidian space of complex dimension $n$. For any two points $z=\left(z_{1}, \ldots, z_{n}\right)$ and $w=\left(w_{1}, \ldots, w_{n}\right)$ in $\mathbb{C}^{n}$ we write $\langle z, w\rangle=z_{1} \bar{w}_{1}+\cdots+z_{n} \bar{w}_{n}$, and $|z|=\sqrt{\langle z, z\rangle}=\sqrt{\left|z_{1}\right|^{2}+\cdots+\left|z_{n}\right|^{2}}$. The set $\mathbb{B}_{n}=\left\{z \in \mathbb{C}^{n}:|z|<1\right\}$ is the open unit ball in $\mathbb{C}^{n}$. Denote by $d v$ the usual Lebesgue volume measure on $\mathbb{B}_{n}$, normalized so that the volume of $\mathbb{B}_{n}$ is one. Throughout this paper we fix a real parameter $\alpha$ with $\alpha>-1$ and write $d v_{\alpha}(z)=c_{\alpha}\left(1-|z|^{2}\right)^{\alpha} d v(z)$, where $c_{\alpha}$ is a positive constant chosen so that $v_{\alpha}\left(\mathbb{B}_{n}\right)=1$.

The weighted Bergman space $A_{\alpha}^{2}\left(\mathbb{B}_{n}\right)$ consists of those functions $f$ holomorphic on $\mathbb{B}_{n}$ that are in the Lebesgue space $L^{2}\left(\mathbb{B}_{n}, d v_{\alpha}\right)$. It is a Hilbert space with inner product

$$
\langle f, g\rangle_{\alpha}=\int_{\mathbb{B}_{n}} f(z) \overline{g(z)} d v_{\alpha}(z) .
$$

The corresponding norm will be denoted by $\|f\|_{\alpha}$. The orthogonal projection $P_{\alpha}$ : $L^{2}\left(\mathbb{B}_{n}, d v_{\alpha}\right) \rightarrow A_{\alpha}^{2}\left(\mathbb{B}_{n}\right)$ is an integral operator given by

$$
P_{\alpha} f(z)=\int_{\mathbb{B}_{n}} \frac{f(w) d v_{\alpha}(w)}{(1-\langle z, w\rangle)^{n+1+\alpha}}, \quad f \in L^{2}\left(\mathbb{B}_{n}, d v_{\alpha}\right) .
$$

Given a finite positive Borel measure $\mu$ on $\mathbb{B}_{n}$, the Toeplitz operator $T_{\mu}$ with symbol $\mu$ acting on $A_{\alpha}^{2}\left(\mathbb{B}_{n}\right)$ is the densely defined integral operator

$$
T_{\mu} f(z)=\int_{\mathbb{B}_{n}} \frac{f(w) d \mu(w)}{(1-\langle z, w\rangle)^{n+1+\alpha}}, \quad f \in A_{\alpha}^{2}\left(\mathbb{B}_{n}\right) .
$$

The operator $T_{\mu}$ is well defined on $H^{\infty}\left(\mathbb{B}_{n}\right)$, the algebra of all bounded holomorphic functions in $\mathbb{B}_{n}$. It is easy to check that $H^{\infty}\left(\mathbb{B}_{n}\right)$ is dense in $A_{\alpha}^{2}\left(\mathbb{B}_{n}\right)$.

For $z \in \mathbb{B}_{n}$, the Berezin transform of $\mu$ is

$$
\widetilde{\mu}(z)=\int_{\mathbb{B}_{n}} \frac{\left(1-|z|^{2}\right)^{n+1+\alpha}}{|1-\langle z, w\rangle|^{2(n+1+\alpha)}} d \mu(w) .
$$

Received by the editors May 12, 2012 and, in revised form, September 4, 2012.

2010 Mathematics Subject Classification. Primary 32A36, 47B10, 47 B35.

Key words and phrases. Bergman spaces, Toeplitz operators, Schatten classes.

The author was supported by SGR grant 2009SGR 420 (Generalitat de Catalunya) and DGICYT grant MTM2011-27932-C02-01 (MCyT/MEC). 
We also need another averaging function. For $z \in \mathbb{B}_{n}$ and $r>0$ define

$$
\widehat{\mu}_{r}(z)=\frac{\mu(D(z, r))}{v_{\alpha}(D(z, r))},
$$

where $D(z, r)=\left\{w \in \mathbb{B}_{n}: \beta(z, w)<r\right\}$ is the Bergman metric ball at $z$ with radius $r$ (here $\beta(z, w)$ denotes the Bergman or hyperbolic metric). The boundedness and compactness of $T_{\mu}$ on $A_{\alpha}^{2}\left(\mathbb{B}_{n}\right)$ for positive $\mu$ can be described using the averaging functions $\widehat{\mu}_{r}$ and $\widetilde{\mu}: T_{\mu}$ is bounded on $A_{\alpha}^{2}\left(\mathbb{B}_{n}\right)$ if and only if $\widetilde{\mu}$ is bounded on $\mathbb{B}_{n}$ if and only if $\widehat{\mu}_{r}$ is bounded on $\mathbb{B}_{n}$, and $T_{\mu}$ is compact on $A_{\alpha}^{2}\left(\mathbb{B}_{n}\right)$ if and only if $\widetilde{\mu} \in C_{0}\left(\mathbb{B}_{n}\right)$ if and only if $\widehat{\mu}_{r} \in C_{0}\left(\mathbb{B}_{n}\right)$ (see [5]). Here $C_{0}\left(\mathbb{B}_{n}\right)$ is the space of all complex-valued continuous functions $f$ on $\mathbb{B}_{n}$ such that $f(z) \rightarrow 0$ as $|z| \rightarrow 1^{-}$.

Concerning the membership of $T_{\mu}$ in the Schatten-Von Neumann ideal $S_{p}$, there is a complete description using the averaging function $\widehat{\mu}_{r}$, but a description using the Berezin transform $\widetilde{\mu}$ can only be obtained for a certain range of $p$. We recall that, for $0<p<\infty$, a compact operator $T$ acting on a separable Hilbert space $H$ belongs to the Schatten class $S_{p}$ if its sequence of singular numbers belongs to the sequence space $\ell^{p}$ (the singular numbers are the square roots of the eigenvalues of the positive operator $T^{*} T$, where $T^{*}$ is the Hilbert adjoint of $T$ ). We refer to [7. Chapter 1] for a brief account on Schatten classes. Part of the result stated below is due to D. Luecking [2] when $n=1$, and the general form is due to K. Zhu [8].

Theorem A. Let $0<p<\infty, r>0$ and $\mu$ be a finite positive Borel measure on $\mathbb{B}_{n}$. Then

(a) $T_{\mu}$ is in $S_{p}$ if and only if $\widehat{\mu}_{r} \in L^{p}\left(\mathbb{B}_{n}, d \lambda\right)$.

(b) If $p>n /(n+1+\alpha)$, then $T_{\mu}$ is in $S_{p}$ if and only if $\tilde{\mu} \in L^{p}\left(\mathbb{B}_{n}, d \lambda\right)$.

Here

$$
d \lambda(z)=\frac{d v(z)}{\left(1-|z|^{2}\right)^{n+1}}
$$

is the Möbius invariant volume measure on $\mathbb{B}_{n}$. The cut-off point $n /(n+1+\alpha)$ in part (b) is best possible [8], and it is related to the finiteness of the measure $\left(1-|z|^{2}\right)^{p(n+1+\alpha)} d \lambda(z)$. One could ask if it is possible to obtain a description in terms of some other integral transform (similar to the Berezin transform) being valid for all $0<p<\infty$. The positive answer given below is the main result of the paper.

Theorem 1. Let $\alpha>-1,0<p<\infty$, and $\mu$ be a finite positive Borel measure on $\mathbb{B}_{n}$. Then $T_{\mu}$ is in the Schatten class $S_{p}$ of $A_{\alpha}^{2}\left(\mathbb{B}_{n}\right)$ if and only if, for each (or some) $t \geq 0$ with $p(n+1+\alpha+2 t)>n$, the function

$$
\left(B^{t} \mu\right)(z)=\int_{\mathbb{B}_{n}} \frac{\left(1-|z|^{2}\right)^{n+1+\alpha+2 t}}{|1-\langle z, w\rangle|^{2(n+1+\alpha+t)}} d \mu(w)
$$

belongs to $L^{p}\left(\mathbb{B}_{n}, d \lambda\right)$.

The necessity of the condition $B^{t} \mu \in L^{p}\left(\mathbb{B}_{n}, d \lambda\right)$ follows easily from Theorem $\mathrm{A}$, so that the interesting case is the sufficiency, especially for small $p$. Throughout the paper, constants are often given without computing their exact values, and the value of a constant $C$ may change from one occurrence to the next.

We need the following well-known integral estimate which has become very important in this area of analysis (see [6, Theorem 1.12], for example). 
Lemma B. Let $t>-1$ and $s>0$. There is a positive constant $C$ such that

$$
\int_{\mathbb{B}_{n}} \frac{\left(1-|w|^{2}\right)^{t} d v(w)}{|1-\langle z, w\rangle|^{n+1+t+s}} \leq C\left(1-|z|^{2}\right)^{-s}
$$

for all $z \in \mathbb{B}_{n}$.

We also need the concept of an $r$-lattice in the Bergman metric. Let $r>0$. A sequence $\left\{a_{k}\right\}$ of points in $\mathbb{B}_{n}$ is called an $r$-lattice if the unit ball is covered by the Bergman metric balls $\left\{D\left(a_{k}, r\right)\right\}$ and if $\beta\left(a_{i}, a_{j}\right) \geq r / 2$ for all $i$ and $j$ with $i \neq j$. If $\left\{a_{k}\right\}$ is an $r$-lattice in $\mathbb{B}_{n}$, then it also has the following property: for any $R>0$ there exists a positive integer $N$ (depending on $r$ and $R$ ) such that every point in $\mathbb{B}_{n}$ belongs to at most $N$ sets in $\left\{D\left(a_{k}, R\right)\right\}$. There are elementary constructions of $r$-lattices in $\mathbb{B}_{n}$.

Proof of the necessity in Theorem 1. The case $p \geq 1($ or $p(n+1+\alpha)>n)$ follows directly from part (b) of Theorem $\mathrm{A}$ since $B^{t} \mu(z) \leq C \widetilde{\mu}(z)$. If $0<p \leq 1$, consider an $r$-lattice $\left\{a_{k}\right\}$. Since $|1-\langle z, w\rangle|$ is comparable to $\left|1-\left\langle z, a_{k}\right\rangle\right|$ for $w \in D\left(a_{k}, r\right)$, we have

$$
\begin{aligned}
\int_{\mathbb{B}_{n}} B^{t} & \mu(z)^{p} d \lambda(z) \\
& \leq \int_{\mathbb{B}_{n}}\left(\sum_{k} \int_{D\left(a_{k}, r\right)} \frac{d \mu(w)}{|1-\langle z, w\rangle|^{2(n+1+\alpha+t)}}\right)^{p}\left(1-|z|^{2}\right)^{p(n+1+\alpha+2 t)} d \lambda(z) \\
& \leq C \int_{\mathbb{B}_{n}}\left(\sum_{k} \frac{\mu\left(D\left(a_{k}, r\right)\right)}{\left|1-\left\langle z, a_{k}\right\rangle\right|^{2(n+1+\alpha+t)}}\right)^{p}\left(1-|z|^{2}\right)^{p(n+1+\alpha+2 t)-n-1} d v(z) \\
& \leq C \sum_{k} \mu\left(D\left(a_{k}, r\right)\right)^{p} \int_{\mathbb{B}_{n}} \frac{\left(1-|z|^{2}\right)^{p(n+1+\alpha+2 t)-n-1} d v(z)}{\left|1-\left\langle z, a_{k}\right\rangle\right|^{2 p(n+1+\alpha+t)}} .
\end{aligned}
$$

Since $p(n+1+\alpha+2 t)>n$ we can apply Lemma $\mathrm{B}$ and the fact that $v_{\alpha}\left(D\left(a_{k}, r\right)\right)$ is comparable to $\left(1-\left|a_{k}\right|^{2}\right)^{n+1+\alpha}$ in order to obtain

$$
\int_{\mathbb{B}_{n}} B^{t} \mu(z)^{p} d \lambda(z) \leq C \sum_{k} \frac{\mu\left(D\left(a_{k}, r\right)\right)^{p}}{\left(1-\left|a_{k}\right|^{2}\right)^{(n+1+\alpha) p}} \leq C \sum_{k} \widehat{\mu}_{r}\left(a_{k}\right)^{p} .
$$

Now, it has already been proved (see [8, p. 305]) that

$$
\sum_{k} \widehat{\mu}_{r}\left(a_{k}\right)^{p} \leq C N \int_{\mathbb{B}_{n}} \widehat{\mu}_{2 r}(z)^{p} d \lambda(z),
$$

and this together with part (a) of Theorem $\mathrm{A}$ finishes the proof of the necessity.

Before going to the proof of the sufficiency we need several properties on reproducing kernels. Recall that $A_{\alpha}^{2}\left(\mathbb{B}_{n}\right)$ is a reproducing kernel Hilbert space with the reproducing kernel function given by

$$
K_{z}^{\alpha}(w)=\frac{1}{(1-\langle w, z\rangle)^{n+1+\alpha}}, \quad z, w \in \mathbb{B}_{n},
$$

with norm $\left\|K_{z}^{\alpha}\right\|_{\alpha}=\sqrt{K_{z}^{\alpha}(z)}=\left(1-|z|^{2}\right)^{-(n+1+\alpha) / 2}$ (see [6]). The normalized kernel functions are denoted by $k_{z}^{\alpha}=K_{z}^{\alpha} /\left\|K_{z}^{\alpha}\right\|_{\alpha}$. If $\left\{e_{k}\right\}$ is any orthonormal set 
of $A_{\alpha}^{2}\left(\mathbb{B}_{n}\right)$, then

$$
\sum_{k}\left|e_{k}(z)\right|^{2} \leq\left\|K_{z}^{\alpha}\right\|_{\alpha}^{2}
$$

with equality if $\left\{e_{k}\right\}$ is an orthonormal basis of $A_{\alpha}^{2}\left(\mathbb{B}_{n}\right)$.

Proof of the sufficiency in Theorem 1: Case $p \geq 1$. Assume that the function $B^{t} \mu$ is in $L^{p}\left(\mathbb{B}_{n}, d \lambda\right)$. In order to show that $T_{\mu}$ is in $S_{p}$, it is enough to see that there is a positive constant $C$ such that

$$
\sum_{k}\left\langle T_{\mu} e_{k}, e_{k}\right\rangle_{\alpha}^{p} \leq C
$$

for all orthonomal sets $\left\{e_{k}\right\}$ of $A_{\alpha}^{2}\left(\mathbb{B}_{n}\right)$ (see [7]). Since

$$
\left\langle T_{\mu} e_{k}, e_{k}\right\rangle_{\alpha}=\int_{\mathbb{B}_{n}}\left|e_{k}(z)\right|^{2} d \mu(z),
$$

the use of the estimate (a consequence of [ [6. Lemma 2.24] and the comparability of the quantities $\left(1-|z|^{2}\right),|1-\langle z, w\rangle|$ and $1-|w|^{2}$ for $\left.w \in D(z, r)\right)$

$$
\left|e_{k}(z)\right|^{2} \leq C \int_{\mathbb{B}_{n}} \frac{\left|e_{k}(w)\right|^{2}\left(1-|w|^{2}\right)^{n+1+\alpha+2 t} d v_{\alpha}(w)}{|1-\langle z, w\rangle|^{2(n+1+\alpha+t)}}
$$

together with Fubini's theorem yields

$$
\left\langle T_{\mu} e_{k}, e_{k}\right\rangle_{\alpha} \leq C \int_{\mathbb{B}_{n}}\left|e_{k}(w)\right|^{2} B^{t} \mu(w) d v_{\alpha}(w) .
$$

Thus, by Hölder's inequality and (1)

$$
\begin{aligned}
\sum_{k}\left\langle T_{\mu} e_{k}, e_{k}\right\rangle_{\alpha}^{p} & \leq C \int_{\mathbb{B}_{n}}\left(\sum_{k}\left|e_{k}(w)\right|^{2}\right) B^{t} \mu(w)^{p} d v_{\alpha}(w) \\
& \leq C \int_{\mathbb{B}_{n}} B^{t} \mu(w)^{p}\left\|K_{w}^{\alpha}\right\|_{\alpha}^{2} d v_{\alpha}(w) \leq C\left\|B^{t} \mu\right\|_{L^{p}\left(\mathbb{B}_{n}, d \lambda\right)}^{p} .
\end{aligned}
$$

In order to deal with the remaining case $0<p<1$ of the sufficiency, we need to introduce some "derivatives" of the kernel functions. For $z, w \in \mathbb{B}_{n}$, define

$$
K_{z}^{\alpha, t}(w)=\frac{1}{(1-\langle w, z\rangle)^{n+1+\alpha+t}}
$$

and let $k_{z}^{\alpha, t}$ denote its normalization, that is, $k_{z}^{\alpha, t}=K_{z}^{\alpha, t} /\left\|K_{z}^{\alpha, t}\right\|_{\alpha}$. Notice that

$$
K_{z}^{\alpha, t}(w)=\overline{R^{\alpha, t} K_{w}^{\alpha}(z)}
$$

where $R^{\alpha, t}$ is the unique continuous linear operator on $H\left(\mathbb{B}_{n}\right)$, the space of all holomorphic functions on $\mathbb{B}_{n}$, satisfying

$$
R^{\alpha, t}\left(\frac{1}{(1-\langle z, w\rangle)^{n+1+\alpha}}\right)=\frac{1}{(1-\langle z, w\rangle)^{n+1+\alpha+t}}
$$

for all $w \in \mathbb{B}_{n}$ (see [7, Section 1.4]). In particular, since $f(z)=\left\langle f, K_{z}^{\alpha}\right\rangle_{\alpha}$ whenever $f \in A_{\alpha}^{2}\left(\mathbb{B}_{n}\right)$, one has

$$
R^{\alpha, t} f(z)=\left\langle f, K_{z}^{\alpha, t}\right\rangle_{\alpha}, \quad f \in A_{\alpha}^{2}\left(\mathbb{B}_{n}\right) .
$$


Here we want to note that

$$
\widetilde{\mu}(z)=\left\langle T_{\mu} k_{z}^{\alpha}, k_{z}^{\alpha}\right\rangle_{\alpha}=\int_{\mathbb{B}_{n}}\left|k_{z}^{\alpha}(w)\right|^{2} d \mu(w),
$$

and, since $\left\|K_{z}^{\alpha, t}\right\|_{\alpha}^{2}$ is comparable to $\left(1-|z|^{2}\right)^{-n-1-\alpha-2 t}$, we have that $B^{t} \mu(z)$ corresponds essentially to the quantity

$$
\left\langle T_{\mu} k_{z}^{\alpha, t}, k_{z}^{\alpha, t}\right\rangle_{\alpha}=\int_{\mathbb{B}_{n}}\left|k_{z}^{\alpha, t}(w)\right|^{2} d \mu(w) .
$$

Taking this into account, the sufficiency for $0<p<1$ will follow from the next lemma. Similar results involving first order derivatives of reproducing kernels appear in [1, 3] and [4].

Lemma 2. Let $T: A_{\alpha}^{2}\left(\mathbb{B}_{n}\right) \rightarrow A_{\alpha}^{2}\left(\mathbb{B}_{n}\right)$ be a positive operator. For $t \geq 0$ set

$$
\widetilde{T^{t}}(z)=\left\langle T k_{z}^{\alpha, t}, k_{z}^{\alpha, t}\right\rangle_{\alpha}, \quad z \in \mathbb{B}_{n}
$$

(a) Let $0<p \leq 1$. If $\widetilde{T^{t}} \in L^{p}\left(\mathbb{B}_{n}, d \lambda\right)$, then $T$ is in $S_{p}$.

(b) Let $p \geq 1$. If $T$ is in $S_{p}$, then $\widetilde{T^{t}} \in L^{p}\left(\mathbb{B}_{n}, d \lambda\right)$.

Proof. Let $p>0$. The positive operator $T$ is in $S_{p}$ if and only if $T^{p}$ is in the trace class $S_{1}$. Fix an orthonormal basis $\left\{e_{k}\right\}$ of $A_{\alpha}^{2}\left(\mathbb{B}_{n}\right)$. Since $T^{p}$ is positive, it belongs to the trace class if and only if

$$
\sum_{k}\left\langle T^{p} e_{k}, e_{k}\right\rangle_{\alpha}<\infty
$$

Let $S=\sqrt{T^{p}}$. Then

$$
\sum_{k}\left\langle T^{p} e_{k}, e_{k}\right\rangle_{\alpha}=\sum_{k}\left\|S e_{k}\right\|_{\alpha}^{2}
$$

and, by [6, Theorem 2.19], this is comparable to

$$
\sum_{k}\left\|R^{\alpha, t} S e_{k}\right\|_{\alpha+2 t}^{2}
$$

Now, by (2), Fubini's theorem and Parseval's identity, we have

$$
\begin{aligned}
\sum_{k}\left\|R^{\alpha, t} S e_{k}\right\|_{\alpha+2 t}^{2} & =\sum_{k} \int_{\mathbb{B}_{n}}\left|R^{\alpha, t} S e_{k}(z)\right|^{2} d v_{\alpha+2 t}(z) \\
& =\sum_{k} \int_{\mathbb{B}_{n}}\left|\left\langle S e_{k}, K_{z}^{\alpha, t}\right\rangle_{\alpha}\right|^{2} d v_{\alpha+2 t}(z) \\
& =\int_{\mathbb{B}_{n}}\left(\sum_{k}\left|\left\langle e_{k}, S K_{z}^{\alpha, t}\right\rangle_{\alpha}\right|^{2}\right) d v_{\alpha+2 t}(z) \\
& =\int_{\mathbb{B}_{n}}\left\|S K_{z}^{\alpha, t}\right\|_{\alpha}^{2} d v_{\alpha+2 t}(z) \\
& =\int_{\mathbb{B}_{n}}\left\langle T^{p} K_{z}^{\alpha, t}, K_{z}^{\alpha, t}\right\rangle_{\alpha} d v_{\alpha+2 t}(z) \\
& =\int_{\mathbb{B}_{n}}\left\langle T^{p} k_{z}^{\alpha, t}, k_{z}^{\alpha, t}\right\rangle_{\alpha}\left\|K_{z}^{\alpha, t}\right\|_{\alpha}^{2} d v_{\alpha+2 t}(z)
\end{aligned}
$$


Putting this all together and taking into account that $\left\|K_{z}^{\alpha, t}\right\|_{\alpha}^{2}\left(1-|z|^{2}\right)^{\alpha+2 t}$ is comparable to $\left(1-|z|^{2}\right)^{-(n+1)}$, we have that $T$ is in $S_{p}$ if and only if

$$
\int_{\mathbb{B}_{n}}\left\langle T^{p} k_{z}^{\alpha, t}, k_{z}^{\alpha, t}\right\rangle_{\alpha} d \lambda(z)<\infty .
$$

Now, both (a) and (b) are deduced from the inequalities (see [7, Proposition 1.31])

$$
\left\langle T^{p} k_{z}^{\alpha, t}, k_{z}^{\alpha, t}\right\rangle_{\alpha} \leq\left[\left\langle T k_{z}^{\alpha, t}, k_{z}^{\alpha, t}\right\rangle_{\alpha}\right]^{p}=\left[\widetilde{T^{t}}(z)\right]^{p}, \quad 0<p \leq 1,
$$

and

$$
\left[\widetilde{T^{t}}(z)\right]^{p}=\left[\left\langle T k_{z}^{\alpha, t}, k_{z}^{\alpha, t}\right\rangle_{\alpha}\right]^{p} \leq\left\langle T^{p} k_{z}^{\alpha, t}, k_{z}^{\alpha, t}\right\rangle_{\alpha}, \quad p \geq 1
$$

This finishes the proof of the lemma.

Proof of the sufficiency in Theorem 1: Case $0<p<1$. Since $B^{t} \mu(z)$ is comparable to $\widetilde{T^{t}}(z)$, the result follows directly from Lemma 2, part (a).

\section{ACKNOWLEDGMENT}

The author would like to thank the referee for valuable comments that improved the final version of the paper.

\section{REFERENCES}

[1] Zen Harper and Martin P. Smith, Testing Schatten class Hankel operators, Carleson embeddings and weighted composition operators on reproducing kernels, J. Operator Theory 55 (2006), no. 2, 349-371. MR2242855 (2007c:47032)

[2] Daniel H. Luecking, Trace ideal criteria for Toeplitz operators, J. Funct. Anal. 73 (1987), no. 2, 345-368, DOI 10.1016/0022-1236(87)90072-3. MR899655 (88m:47046)

[3] J. Pau and J.A. Peláez, Schatten classes of integration operators on Dirichlet spaces, J. Anal. Math. 120 (2013), 255-289. MR3095154

[4] M. P. Smith, Testing Schatten class Hankel operators and Carleson embeddings via reproducing kernels, J. London Math. Soc. (2) 71 (2005), no. 1, 172-186, DOI 10.1112/S0024610704005988. MR2108255(2005h:47056)

[5] Ke He Zhu, Positive Toeplitz operators on weighted Bergman spaces of bounded symmetric domains, J. Operator Theory 20 (1988), no. 2, 329-357. MR1004127 (92f:47022)

[6] Kehe Zhu, Spaces of holomorphic functions in the unit ball, Graduate Texts in Mathematics, vol. 226, Springer-Verlag, New York, 2005. MR2115155 (2006d:46035)

[7] Kehe Zhu, Operator theory in function spaces, 2nd ed., Mathematical Surveys and Monographs, vol. 138, American Mathematical Society, Providence, RI, 2007. MR2311536 (2008i:47064)

[8] Kehe Zhu, Schatten class Toeplitz operators on weighted Bergman spaces of the unit ball, New York J. Math. 13 (2007), 299-316. MR2357717 (2008g:47058)

Departament de Matemàtica Aplicada i Analisi, Universitat de Barcelona, Gran Via 585, 08007 BARCELONA, SPAIN

E-mail address: jordi.pau@ub.edu 